\title{
Acute Myocardial Infarction due to Left Atrial Myxoma
}

\author{
Shimpei Ito ${ }^{1}$, Akihiro Endo ${ }^{1}$, Taiji Okada ${ }^{1}$, Taku Nakamura ${ }^{1}$, Tomoko Adachi ${ }^{1}$, \\ Ryuma Nakashima ${ }^{1}$, Takashi Sugamori ${ }^{1}$, Nobuyuki Takahashi ${ }^{1}$, \\ Hiroyuki Yoshitomi ${ }^{2}$ and Kazuaki Tanabe ${ }^{1}$
}

\begin{abstract}
Myxoma is a common benign cardiac tumor that may rarely cause an acute myocardial infarction. A 77year-old woman was admitted to our hospital with chest pain. Electrocardiography showed an ST elevation in leads $\mathrm{V}_{3-6}$. Transthoracic echocardiography revealed an ovoid mass with fragmentation in the left atrium and hypokinesia of the left ventricular apex. Coronary angiography indicated the presence of a coronary embolism that was suspected to be from the left atrial mass. The mass was removed by emergency surgical resection to avoid a further systemic embolism and was diagnosed pathologically as a myxoma. The patient was discharged after 13 days with no complications.
\end{abstract}

Key words: myocardial infarction, myxoma

(Intern Med 55: 49-54, 2016)

(DOI: 10.2169/internalmedicine.55.5179)

\section{Introduction}

Myxoma, a rare condition, is the most common type of primary cardiac tumor, and accounts for half of all cases of benign cardiac tumors (1). Despite its benign pathologic nature, catastrophic results can occur as a result of systemic embolism, including myocardial infarction. In this report, we describe a case of acute myocardial infarction caused by coronary embolism that was suspected to be due to a left atrial (LA) myxoma.

\section{Case Report}

A 77-year-old woman without any coronary risk factors other than hypertension was admitted to the emergency department of our hospital with chest pain. She had not previously noticed any chest pain or dyspnea on exertion; however, her body weight had decreased by $3 \mathrm{~kg}$ in three months. On physical examination, the patient's blood pressure was 152/97 $\mathrm{mmHg}$, her heart rate was 84 beats/min, her oxygen saturation was $95 \%$ with room air, her respiratory rate was 12 breaths/min, and her body temperature was $36.7^{\circ} \mathrm{C}$. Auscultation revealed a systolic murmur (Levine $2 /$ 6) at the level of the third left intercostal space and no lung rales; moreover, no rashes or lymphadenopathy were noted. Her cardiothoracic ratio, measured on a chest X-ray, was $60 \%$. An electrocardiogram showed ST elevation in leads $\mathrm{V}_{3-6}$ (Fig. 1). The laboratory findings included a cardiac troponin I level of $3.31 \mathrm{ng} / \mathrm{mL}$, a creatine kinase (CK) level of $210 \mathrm{U} / \mathrm{L}$, and a CK-MB level of $20.9 \mathrm{U} / \mathrm{L}$. The patient's white blood cell count was $4,610 / \mu \mathrm{L}$ and her D-dimer level was $3.4 \mu \mathrm{g} / \mathrm{mL}$. Moreover, her $\mathrm{C}$ reactive protein level was $1.3 \mathrm{mg} / \mathrm{mL}$, her erythrocyte sedimentation rate was $87 \mathrm{~mm} /$ $\mathrm{h}$, and her B-type natriuretic peptide level was $30.1 \mathrm{pg} / \mathrm{mL}$.

A transthoracic echocardiographic (TTE) examination showed a left ventricular diastolic diameter of $42 \mathrm{~mm}$, a systolic diameter of $28 \mathrm{~mm}$, and an ejection fraction of 61\%. The LA was dilated (LA diameter: $36 \mathrm{~mm}$, LA volume index: $37 \mathrm{~mL} / \mathrm{m}^{2}$ ), although the mitral inflow pattern was consistent with the patient's age (E wave peak: $70 \mathrm{~cm} /$ $\mathrm{s}$; A wave peak: $102 \mathrm{~cm} / \mathrm{s}$; E deceleration time: $248 \mathrm{~ms}$ ). Hypokinesia of the left ventricular apex was noted. The TTE examination also revealed a mobile mass in the LA, although there was no evidence to suggest it was obstructing the mitral valve (Fig. 2).

${ }^{1}$ Division of Cardiology, Shimane University Faculty of Medicine, Japan and ${ }^{2}$ Clinical Laboratory Department, Shimane University Hospital, Japan

Received for publication February 17, 2015; Accepted for publication April 14, 2015

Correspondence to Dr. Shimpei Ito, shimpei@med.shimane-u.ac.jp 


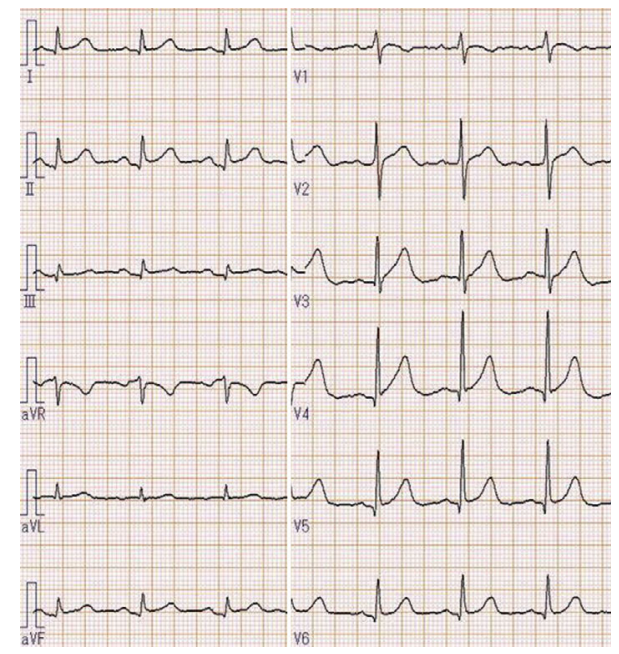

Figure 1. An electrocardiogram showing ST elevation in leads $\mathbf{V}_{3-6}$.
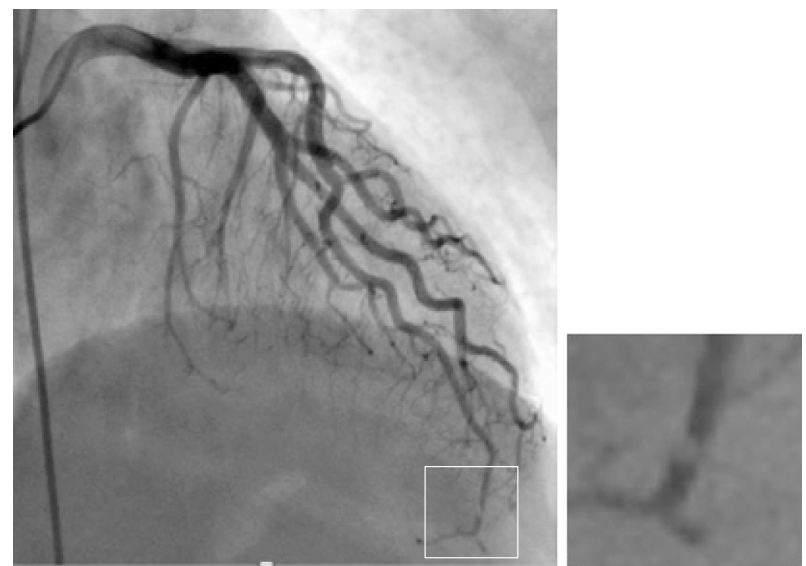

Figure 3. Coronary angiography indicated sub-total occlusion of the distal left anterior descending artery (box frame), which was suspected to be due to an embolism caused by the left atrial mass.

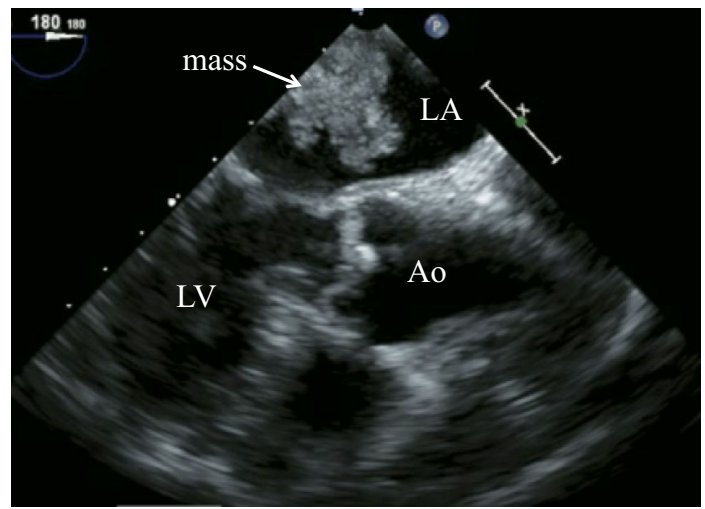

Figure 5. Transesophageal echocardiography showed a mobile mass, $31 \times 27 \mathrm{~mm}$ in size, with an irregular and fernlike surface and an inhomogeneous appearance (arrow). LA: left atrium, LV: left ventricle, Ao: aorta

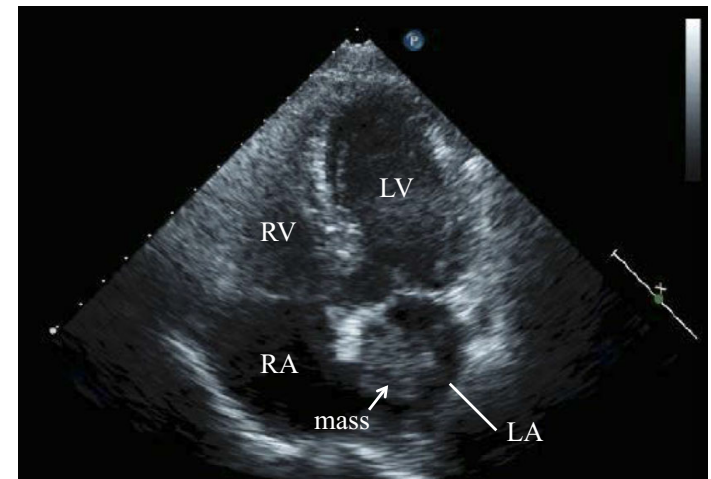

Figure 2. Transthoracic echocardiography showed hypokinesia of the left ventricular apex and revealed a mobile mass in the left atrium (arrow). LV: left ventricle, RV: right ventricle, LA: left atrium, RA: right atrium

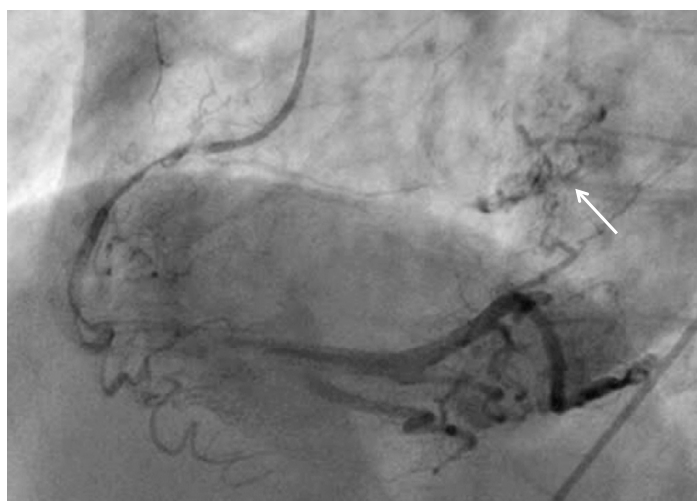

Figure 4. Coronary angiography also revealed the presence of feeding arteries toward the left atrial mass from the right coronary artery (arrow).

Because acute anterior myocardial infarction was suspected, we performed emergency coronary angiography (CAG). CAG indicated the sub-total occlusion of the distal left anterior descending artery (LAD), which was suspected to be due to an embolism from the left atrial mass (Fig. 3). In addition, CAG indicated the presence of feeding arteries toward the LA mass from the right coronary artery (RCA) (Fig. 4). The LA mass was swinging in the heart chambers during the cardiac cycle. Percutaneous coronary interventions, such as aspiration for coronary embolism, were not performed because the culprit lesion was too distal.

Right heart catheterization revealed that the patient's hemodynamic status was within normal limits. After CAG, transesophageal echocardiography (TEE) was performed, which showed a mobile mass, measuring $31 \times 27 \mathrm{~mm}$, with an irregular and fernlike surface and an inhomogeneous appearance (Fig. 5). The mass was widely attached to the interatrial septum between the fossa ovalis and the mitral valve. Color Doppler signals were detected in the mass. Cardiac magnetic resonance imaging (MRI) demonstrated that the mass originated in the left atrium. The signal intensity of the mass was similar to that of the myocardium on T1weighted images, and high on T2-weighted images, along 

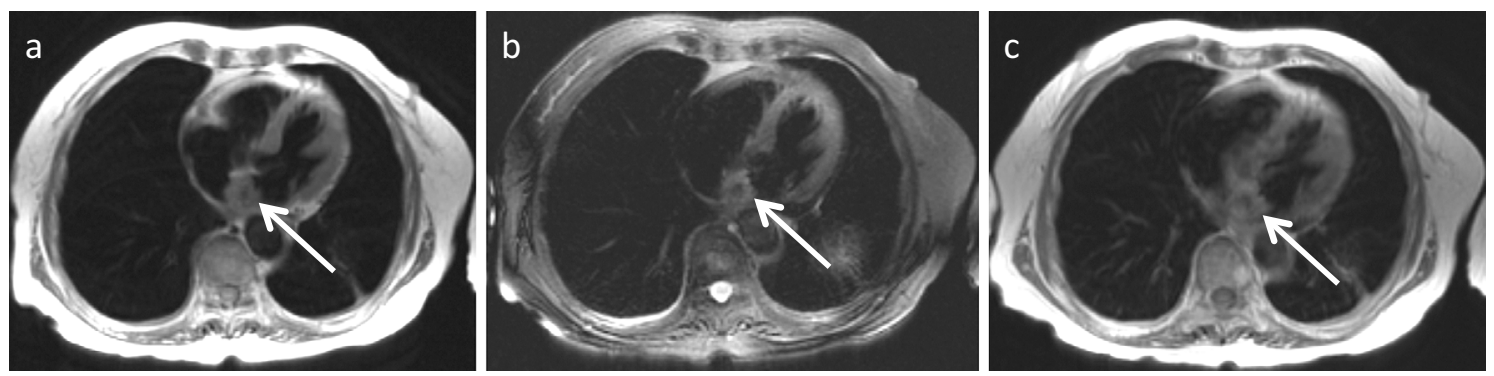

Figure 6. Cardiac magnetic resonance imaging demonstrated that the mass originated in the left atrium (arrows). The signal intensity of the mass was similar to that of the myocardium on T1-weighted images (a), and high on T2-weighted images (b), along with enhancement on gadolinium-enhanced images (c).
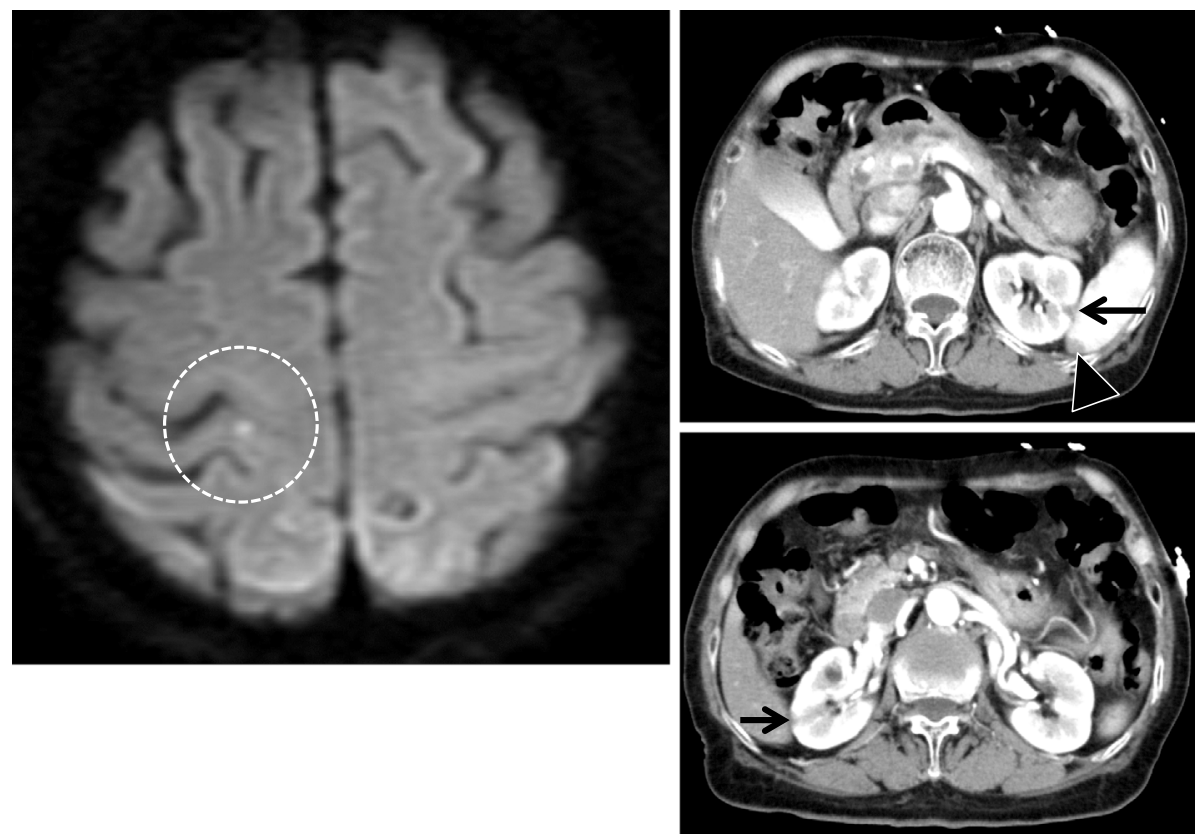

Figure 7. Magnetic resonance imaging of the head indicated a small cerebral infarction (white dotted circle). Enhanced computed tomography indicated infarctions of both the kidney (black arrows) and the spleen (black arrowhead).

with enhancement on gadolinium-enhanced images (Fig. 6). These findings on cardiac MRI are typical findings for myxoma. Moreover, MRI of the head detected a small cerebral infarction, while both kidney and spleen infarctions were detected by enhanced computed tomography (Fig. 7). The patient's peak CK and CK-MB were 382 U/L and 30.1 $\mathrm{U} / \mathrm{L}$ on day 2 , respectively. With respect to electrocardiogram, $\mathrm{ST}$ elevation in leads $\mathrm{V}_{3-6}$ changed to terminal $\mathrm{T}$ wave inversion on day 2 and upright $\mathrm{T}$ waves on day 4 (Fig. 8).

Emergency surgical resection of the villous mass was performed to avoid further systemic embolism. The histopathological examination of the left atrial mass determined that it was a myxoma, and also demonstrated the presence of bleeding (Fig. 9). ${ }^{99 \mathrm{~m}}$ Technetium pharmacological stress myocardial perfusion imaging showed no defect on either the stress or the rest image after surgery (Fig. 10). There were no complications in the postoperative period. A TTE examination performed one week after surgery, showed an ejection fraction of $66 \%$ without asynergy. The patient was discharged after 13 days.

\section{Discussion}

Primary cardiac tumors are rare, with a frequency of only $0.0017-0.033 \%$ in autopsy cases (2). Myocardial infarction due to myxoma is also extremely rare. None of the 74 cases of coronary emboli analyzed in a retrospective autopsy study was due to a myxoma (3). The incidence of coronary embolization due to cardiac myxoma is reported to be only $0.06 \%$ (4). According to a review of 40 cases of coronary artery embolization secondary to atrial myxoma, the patients' mean age was approximately 50 years, the RCA was a common culprit, and inferior myocardial infarction was seen in most cases (5). Panos et al. investigated the lesions and culprit arteries of myocardial infarctions due to cardiac myxomas that were diagnosed by echocardiography (6). In 


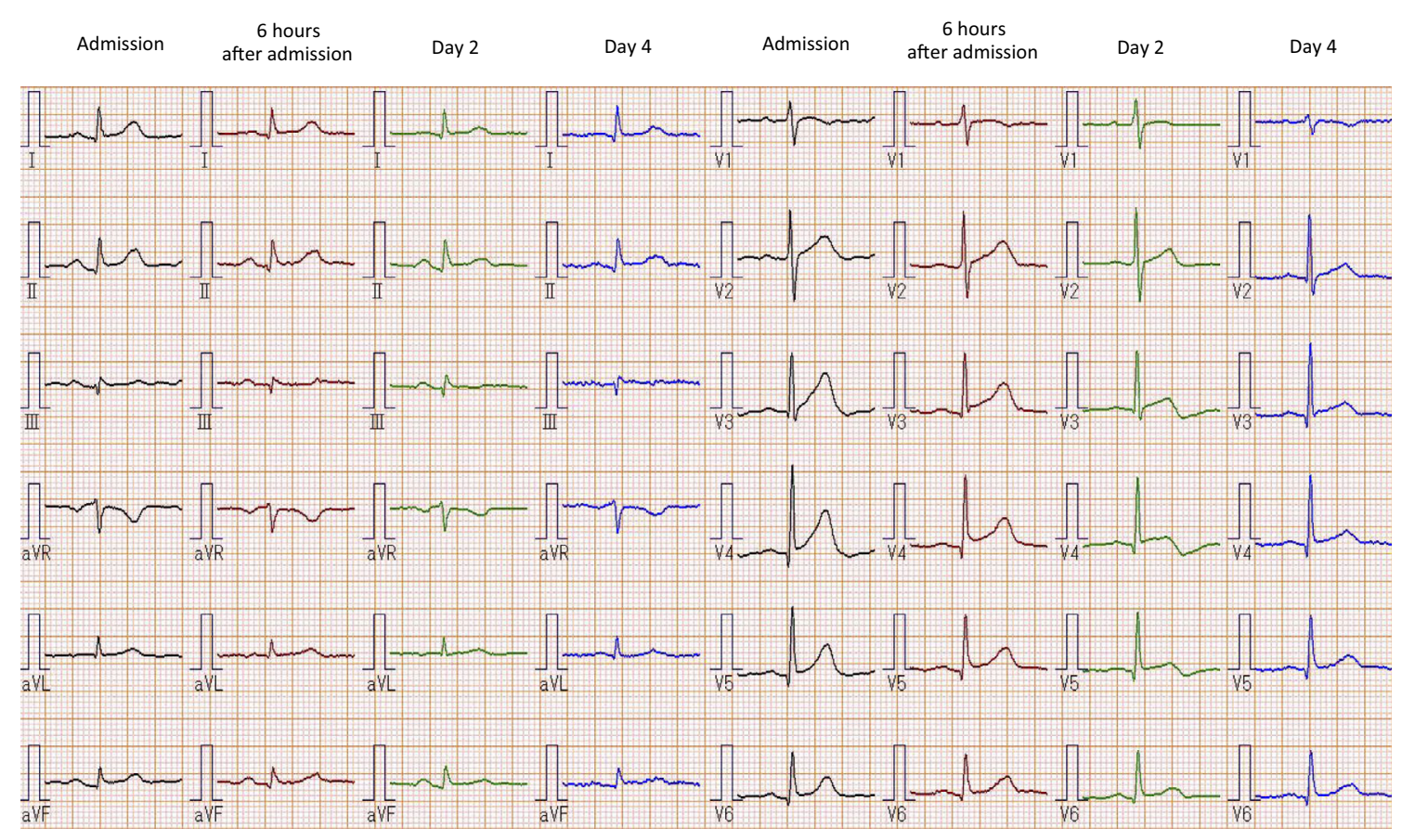

Figure 8. Electrocardiograms were recorded on admission, six hours after admission, on day 2 and on day 4. The $S T$ elevation in leads $V_{\text {3-6 }}$ changed to terminal $T$ wave inversion on day 2 and upright $T$ waves on day 4 .
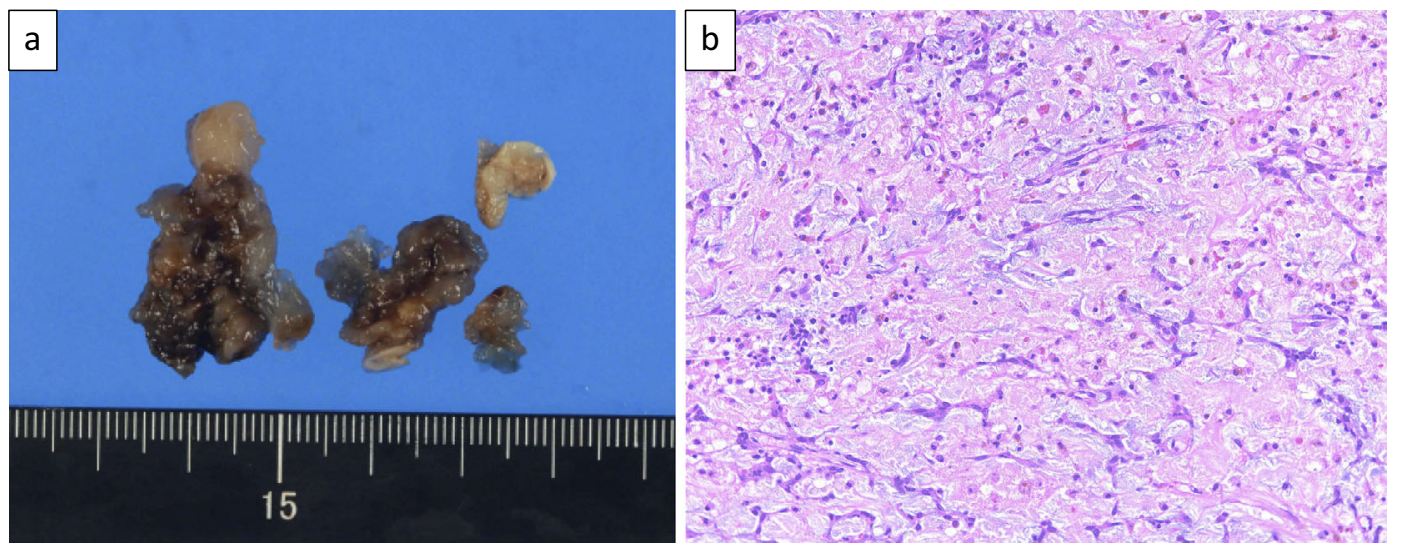

Figure 9. a: The villous mass after surgical resection. b: The histological findings, which were typical of myxoma.

their report, inferior myocardial infarctions were seen in $63.3 \%$, anterior infarctions were noted in $22.7 \%$, and posterior infarctions were observed in $9.1 \%$ of the cases. CAG showed RCA embolization in $47.6 \%$ of the patients with embolism, and the investigators speculated that the reason for this high frequency was the anatomical position of the RCA ostium in relation to the aortic blood flow (6). They also reported that $23.8 \%$ of the cases had a normal angiogram. Braun et al. noted that the coronary artery may still appear to be normal, despite a clinical diagnosis of acute embolic myocardial infarction, because of the myxomatous histology of the tumor, which allows it to embolize and resolve spontaneously (5). Ibrahim et al. reviewed 17 cases of myocardial infarction due to LA myxoma from 2003 to 2013 (7). According to the review, 10 case reports showed no evidence of myocardial infarction on CAG. Our patient was diagnosed with myocardial arterial embolism in the $\mathrm{LAD}$, as detected by CAG. We considered our case to involve acute LAD myocardial infarction due to embolization from the myxoma, based on the CAG and echocardiography findings and the absence of any other atherosclerotic risk factors (with the exception of hypertension).

Systemic embolism due to atrial myxoma is frequently noted in the clinical setting; in one study, this condition occurred in $32 \%$ of cases as the presenting symptom (8). However, the incidence of coronary embolization due to atrial myxoma is only $0.06 \%$. Lehrman et al. suggested that a possible explanation for this low incidence could involve the right-angled junction of the coronary apertures within the aortic root, the protection of the coronary arteries by the 


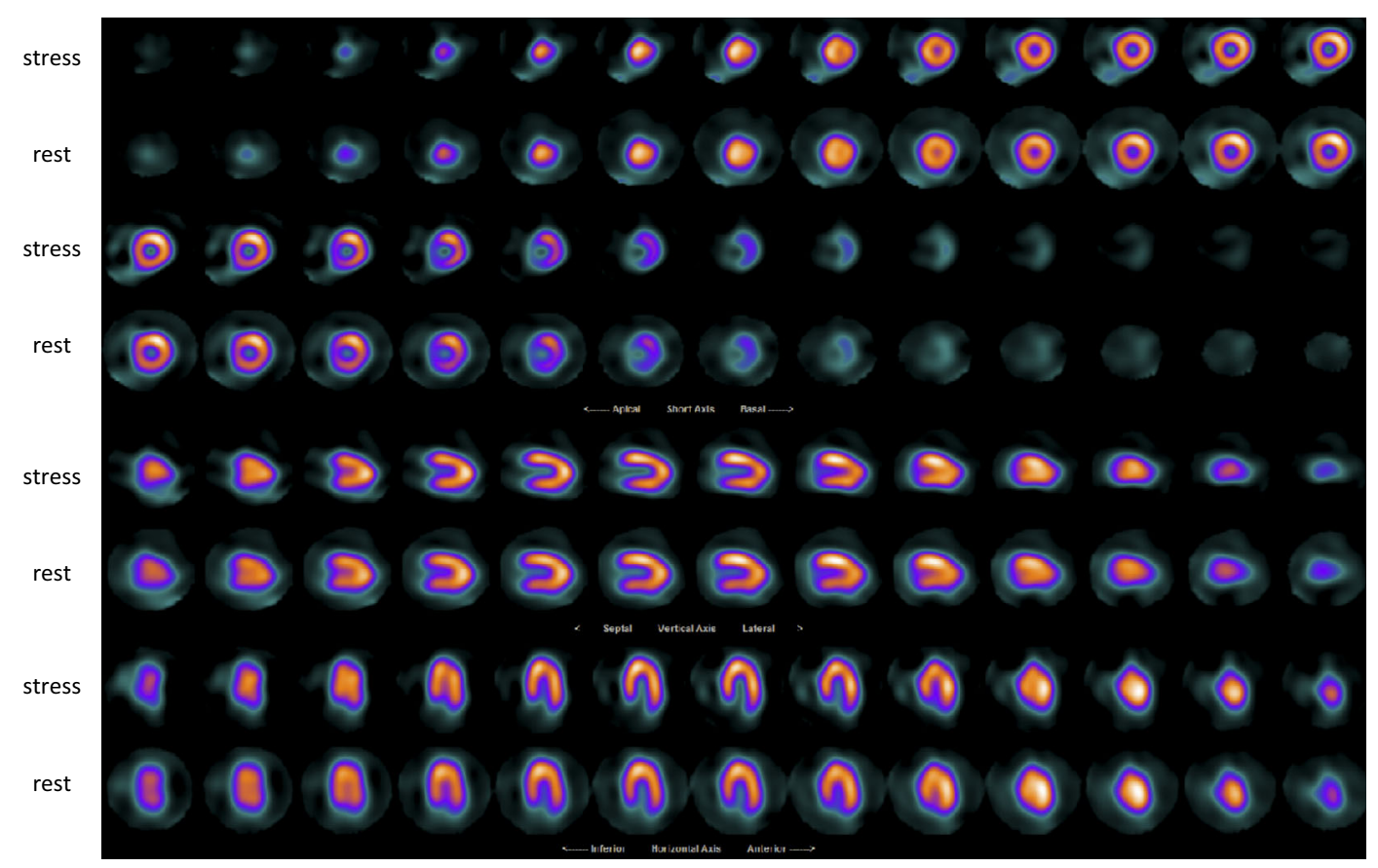

Figure 10. ${ }^{99}$ mechnetium pharmacological stress myocardial perfusion imaging showed no defect on either the stress or the rest image after surgery.

aortic valve cusps, the small diameter of the apertures, and the filling of the coronary arteries during ventricular diastole (4). Although less common, myxoma has been reported to cause death from coronary embolization (9). Thus, it is clinically important to identify the patients who are at a high risk of systemic embolism caused by cardiac myxoma.

The early detection of cardiac myxoma and the assessment of the risk of systemic embolism are important for the prevention of serious complications, including fatal myocardial infarction. Echocardiography is widely available and is often utilized as a diagnostic test in patients with a typical symptom of myxoma. Two different anatomic types of myxoma have been determined through echocardiography: round, which is solid and has a round shape with a nonmobile surface; and polypoid, which is soft and has an irregular shape with a mobile surface (10). The incidence of systemic embolism is higher in tumors with an irregular and friable surface than in those with a smooth surface (11). Ha et al. showed that prolapsing and polypoid tumors were associated with embolism (10). The more irregular and friable the myxomas, the higher the likelihood of embolism. Another study reported that the incidence of neurologic symptoms and brain infarctions was significantly higher for papillary-type myxoma, which is soft and has an irregular shape, than for the ovoid type myxoma, which is solid and has a smooth surface (12). In our patient, the appearance of the tumor was irregular; it had a mobile surface with fragmentations, which indicated a high risk of systemic embolization. Hence, emergent surgical resection was subsequently performed.

Echocardiography should be performed in detail as soon as possible to guide the management of patients with car- diac myxoma. Although most LA myxomas are attached to the LA septum, Swartz et al. reported that an extraseptal location was correlated with embolic events (13). It is also useful to assess the risk of embolism with findings on CAG. Shimono et al. found that clusters of tortuous tumor vessels on CAG may predict a solid type tumor (14). On the other hand, a lack of tortuous vessels was identified in papillarytype myxomas. In our patient, given that contrast medium pooling was only identified by CAG in the feeding artery, the myxoma was judged to be of the papillary type, with a high risk of embolization.

Our patient had lost weight, which is considered to be one of the constitutional symptoms of myxoma. It is reported that constitutional symptoms are mediated through increased interleukin-6 (IL-6) release from myxoma cells (15). In the present case, the preoperative IL-6 level was $10.77 \mathrm{pg} / \mathrm{mL}$. Wada et al. reported a case of cardiac myxoma that metastasized to the brain and suggested that IL-6 might possibly potentiate metastasis of a cardiac myxoma (16). Therefore, our myxoma patients might be at a higher risk of coronary embolism. Moreover, the progression of myocardial ischemia is possible due to the metastasis of a myxoma to a coronary artery. In such cases it is important to assess the progression of myocardial ischemia with coronary computed tomography, myocardial perfusion imaging, or coronary angiography, in addition to performing echocardiography at periodic intervals after the resection of the tumor.

We herein reported an interesting case of myocardial infarction due to coronary embolization. Coronary embolization secondary to atrial myxoma is rare. In this case, the embolization was judged to be due to the presence of an 
atrial myxoma, based on the findings of CAG and echocardiography. Thus, it is important to assess the risk of systemic embolization as soon as possible when an atrial myxoma is identified, in order to guide the effective management of such patients.

The authors state that they have no Conflict of Interest (COI).

\section{References}

1. McAllister HA Jr, Hall RJ, Cooley DA. Tumors of the heart and pericardium. Curr Probl Cardiol 24: 57-116, 1999.

2. Lam KY, Dickens P, Chan AC. Tumors of the heart. A 20-year experience with a review of 12,485 consecutive autopsies. Arch Pathol Lab Med 117: 1027-1031, 1993.

3. Wenger NK, Bauer S. Coronary embolism. Am J Med 25: 549$557,1958$.

4. Lehrman KL, Prozan G, Ullyot D. Atrial myxoma presenting as acute myocardial infarction. Am Heart J 110: 1293-1295, 1985.

5. Braun S, Schrotter H, Reynen K, Schwencke C, Strasser RH. Myocardial infarction as complication of left atrial myxoma. Int $\mathbf{J}$ Cardiol 101: 115-121, 2005.

6. Panos A, Kalangos A, Sztajzel J. Left atrial myxoma presenting with myocardial infarction. Case report and review of the literature. Int J Cardiol 62: 73-75, 1997.

7. Al Zahrani IM, Alraqtan A, Rezk A, Almasswary A, Bella A. Atrial myxoma related myocardial infarction: case report and review of the literature. J Saudi Heart Assoc 26: 166-169, 2014.
8. Gelder HM, Jacobs JP, McCormack J. Acute myocardial infarction in a 15-year old secondary to myxomatous embolisation. Cardiol Young 14: 658-660, 2004.

9. Morais C, Falzoni R, Alves VA. Myocardial infarct due to a unique atrial myxoma with epithelial-like cells and systemic metastases. Arch Pathol Lab Med 112: 185-190, 1988.

10. Ha JW, Kang WC, Chung N, et al. Echocardiographic and morphologic characteristics of left atrial myxoma and their relation to systemic embolism. Am J Cardiol 83: 1579-1582, 1999.

11. Goswami KC, Shrivastava S, Bahl VK, Saxena A, Manchanda SC, Wasir HS. Cardiac myxomas: clinical and echocardiographic profile. Int J Cardiol 63: 251-259, 1998.

12. St John Sutton MG, Mercier LA, Guliani ER, Lie JT. Atrial myxoma: a review of clinical experience in 40 patients. Mayo Clin Proc 55: 371-376, 1980.

13. Swartz MF, Lutz CJ, Chandan VS, Landas S, Fink GW. Atrial myxomas: pathologic types, tumor location, and presenting symptoms. J Card Surg 21: 435-440, 2006.

14. Shimono T, Makino S, Kanamori Y, Kinoshita T, Yada I. Left atrial myxomas: using gross anatomic tumor types to determine clinical fratures and coronary angiographic findings. Chest 107: 674-679, 1995.

15. Kamata S, Kawada T, Kikuchi K, et al. Clinical analysis of embolism with left atrial myxoma. Kyobu Geka (The Japanese Journal of Thoracic Surgery) 49: 297-300, 1996 (in Japanese, Abstract in English).

16. Wada A, Kanda T, Hayashi R, Imai S, Suzuki T, Murata K. Cardiac myxoma metastasized to the brain: potential role of endogenous interleukin-6. Cardiology 83: 208-211, 1993.

(C) 2016 The Japanese Society of Internal Medicine http://www.naika.or.jp/imonline/index.html 